\title{
A hydrogel based quasi-stationary test system for in vitro dexamethasone release studies for middle ear drug delivery systems
}

\begin{abstract}
For the investigation of in vitro drug release, methods have been used in which samples of drug delivery systems are immersed in release medium. The medium is used to measure drug concentration via chromatography or photometry. These systems are suitable to investigate the drug release of different systems or to simulate tissue environments. When considering predominantly humid regions, e.g. for drug release into the cochlea through the round window membrane by a drug delivery system placed at that membrane, reproducible in vitro determination of drug release becomes particularly challenging. In this study the development of a system is reported that allows the investigation of the in vitro drug release simulating such conditions.
\end{abstract}

The presented test system consists of an alginate hydrogel in glass vials simulating the biological membrane, which separates the drug delivery system from the medium filled compartment. Saline is used as release medium and injected under the hydrogel. The samples are placed on top of the hydrogel, which slightly contacts the medium surface. The drug concentration in the release medium was determined by HPLC measurements.

This system allows for testing the release of dexamethasone without the samples being completely surrounded by medium. The hydrogel mediates the diffusion of the drug by ensuring the contact with the medium. Release was monitored for more than 23 days.

The presented concept was successfully designed and manufactured. The system is inexpensive and can be duplicated easily. In this study, it was used to monitor the drug release of dexamethasone from PEGDA $_{700}$ derived polymer. One challenge that remains to be considered is

\footnotetext{
*Corresponding author: Eickner Thomas: Institute for Biomedical Engineering (University Medical Center Rostock, University of Rostock), Friedrich-Barnewitz-Straße 4, Rostock, Germany, thomas.eickner@uni-rostock.de

Michael Teske, Natalia Rekowska, Volkmar Senz, Niels Grabow, Institute for Biomedical Engineering (University Medical Center Rostock, University of Rostock), Rostock, Germany, Klaus-Peter Schmitz, University Medical Center Rostock, and Institute for ImplantTechnology and Biomaterials e.V, Warnemünde, Germany,
}

the low mechanical stability of the hydrogel, which results in a need for repeated manufacturing during the handling of the system.

Keywords: drug release, dexamethasone, PEGDA, alginate, https://doi.org/10.1515/cdbme-2021-2175

\section{Introduction}

In vitro drug release methods usually work by immersing samples of drug delivery systems (DDS) in release medium at agitation and defined temperatures. DDS usually consists of one or more active pharmaceutical ingredients that are immobilized in a polymer matrix or on the surface of such material. The release medium serves as solvent for the drug and subsequently allows measuring the concentration via chromatographical or photometrical methods. Thereby the drug release depends on different parameters, mainly the surface to volume ratio of the DDS. These in vitro test systems are suitable to investigate different types of DDS concerning reproducibility and comparison also for cochlear drug delivery [1]. They can be also used to simulate drug release in tissues that are filled with liquids [2]. When considering predominantly humid regions, e.g. the middle ear, reproducible in vitro determination of drug release becomes particularly challenging. Whereas an immersion of DDS in release medium ensures the drug diffusion, over the whole surface, humid regions may facilitate only a partial surface of the DDS, e.g. for DDS that are placed in the middle ear at the round window membrane to release drugs into the perilymph of the cochlea by allowing the drug to diffuse through that membrane. These circumstances lead to additional parameters that may lead to higher variances in drug release. In this study the development of a quasi-stationary system is reported, that allows adjustment in size and volume, the sampling at regular intervals and determining the drug concentration.

There are several conditions to be met for such system.

- It should separate the DDS from the medium compartment, while ensuring the contact between both.

- The release medium has to be able to solve the released drug. 
- The production of such a system should be fast

- A repeated medium change should be possible.

- The gel should not interfere with the release.

The system reported here makes use of widely used laboratory materials and an alginate hydrogel to mediate the diffusion of the drug from the DDS into the release medium by ensuring the contact between both. Alginate allows an easy production of test systems and withstands a number of medium changes. Isotonic saline was used as release medium.

DDS test samples were made of PEGDA mixed with dexamethasone. PEGDA is a material of interest for deriving biocompatible polymers by additive manufacturing procedures such as stereolithography. This material may therefore be a candidate for DDS for the round window membrane fulfilling the need for highly individualized geometries in the middle ear.

\section{Materials and methods}

\subsection{Preparation of Dexamethasone- PEGDA Samples}

PEGDA $_{700}(\mathrm{Mw} \sim 700 \mathrm{~g} / \mathrm{mol}$, Sigma-Aldrich, CAS: 2657048-9) was mixed with water $(1: 1 \mathrm{v} / \mathrm{v})$, Irgacure (SigmaAldrich, CAS: 106797-53-9) stock solution (c=500 mg/mL in dimethylsulfoxid (DMSO) (Sigma-Aldrich, CAS: 67-68-5) in a ratio of $39: 1 \mathrm{v} / \mathrm{v}$ and dexamethasone (Sigma-Aldrich, CAS: $50-02-2$ ) in a ratio of $15 \%$ according to the mass of PEGDA $_{700}$.

The mixture was pipetted into moulds with a volume of $30 \mu \mathrm{L}$ each. The samples were polymerized for $30 \mathrm{~min}$ in the UV chamber CL-1000L (UVP, USA) at $\lambda=365 \mathrm{~nm}$. The resulting polymer discs had a diameter of $5 \mathrm{~mm}$. They were dried overnight at $40^{\circ} \mathrm{C}$ in vacuum and weighed afterwards on a KERN 770-60 balance. Discs with comparable masses $(\mathrm{m}=17.27 \pm 0.13 \mathrm{mg})$ were chosen.

\subsection{Concept and Design}

The concept (Fig. 1a) provides a fluid-filled compartment (Fig. 1a, light blue) that is covered by a hydrogel (Fig. 1a, light green). The fluid serves as the appropriate release medium, while the hydrogel serves as the platform for the drug release system (Fig. 1a, dark green). In order to change the release medium at defined time points by aspiration of the medium with a syringe, the system needs pressure compensation between the medium compartment and the surrounding air, which is ensured by a short cannula. Otherwise the hydrogel would be pressed to the bottom of the vial by air pressure or it would break during the medium change. a)

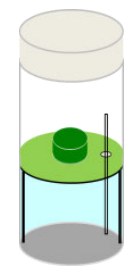

b)

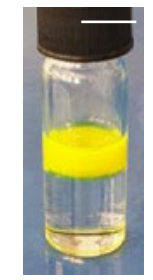

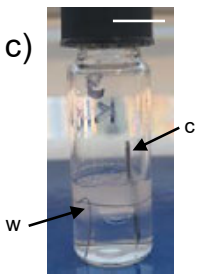

Figure 1: a) Concept for the test system; a glass vial (grey) equipped with release medium (light blue) under a hydrogel layer (light green), a cannula for pressure compensation (black rod) and a wire rack (black). The DDS (dark green) is placed on the hydrogel layer b) Illustrative example of the system without a cannula and wire rack, the hydrogel has been stained with fluorescein for better visibility. c) test system including a cannula (c) for pressure compensation and a wire rack (w) to prevent the hydrogel from sinking, Scale bars (white) correspond to $1 \mathrm{~cm}$.

\subsection{Preparation of the in vitro test system}

The in vitro test system was prepared directly in a $4 \mathrm{~mL}$ glass vial (outer diameter $1.5 \mathrm{~cm}$ ), which would subsequently also be used for the drug release testing. For this purpose, a wire rack was placed in the vial (Fig. 1c) to prevent the alginate hydrogel from sinking into the release medium.

For preparation of the alginate hydrogel the following solutions were prepared according to [3]: Solution A: 3\% sodium alginate (Sigma-Aldrich, CAS: 9005-38-3) (in water, Solution B: $0.15 \mathrm{~g} \mathrm{CaSO}_{4} * 2 \mathrm{H}_{2} \mathrm{O}$ in $2 \mathrm{~g}$ of a $2.5 \%$ sodium triphosphate solution in water.

The vial was filled with $2 \mathrm{ml}$ chloroform $\left(\mathrm{CHCl}_{3}\right.$ J.T.Baker CAS: 67-66-3) completely covering the wire rack. Solution B was then mixed with $16.5 \mathrm{~g}$ solution A and pipetted dropwise using a $1 \mathrm{~mL}$ Eppendorf pipette onto the $\mathrm{CHCl}_{3}$-surface until it was completely covered. Afterwards the gel was allowed to cure for $15 \mathrm{~min}$. A cannula was shortened and pierced through the gel in order to connect the medium compartment under the hydrogel and the air filled compartment over it, and remains in the system for pressure compensation during the medium change. The $\mathrm{CHCl}_{3}$ is removed by the means of a syringe and the medium compartment was rinsed three times by injecting and aspirating isotonic saline $(0.9 \% \mathrm{NaCl})$ under the alginate. For completion, the volume under the hydrogel was filled with isotonic saline $(2 \mathrm{~mL})$. 


\subsection{In vitro drug release}

The polymer-drug discs were positioned on the hydrogel surface and stored at $37^{\circ} \mathrm{C}$ without shaking. After defined periods of time, the medium was exchanged using a syringe and diluted 1:10 prior to the HPLC measurements. Samples were taken on days: $1,3,5,7,10,13,17,18,19,20,21,22$, 23 and 24. Quantification of dexamethasone was performed on an HPLC system (Knauer, Berlin, Germany) equipped with a Chromolith FastGrad RP-18e 50-2 column. Methanol/Water 1:1 was used as mobile phase. Detection occurred with a UV-Detector at the wavelength $\lambda=254 \mathrm{~nm}$ [2].

\section{Results}

It was possible to determine concentrations of dexamethasone in the medium, without the samples being completely surrounded by medium (Fig. 2). Cumulative release of dexamethasone resulted in $490 \pm 60.3 \mu \mathrm{g}$ after $24 \mathrm{~d}$ with a calculated initial loading of $2.65 \pm 0.02 \mathrm{mg}$ in the DDS.

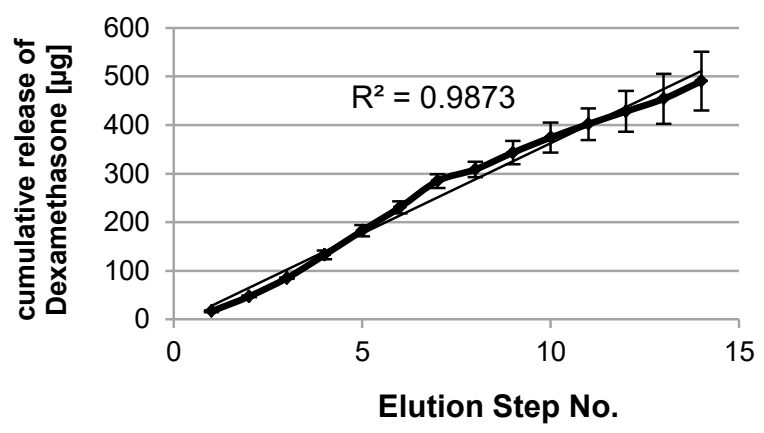

Figure 2: Cumulative release of dexamethasone $(n=3)$, the linearity to elution step number is explained by the quasistationary system that does not ensure sink conditions, Coefficient of determination $\left(R^{2}\right)$ is shown in the diagram, standard deviation is indicated by error bars.

The linearity to elution step number is explained by the quasi-stationary system that does not ensure sink conditions. The hydrogel mediates the contact between DDS and release medium and hence mediates the diffusion of the drug. Drug concentration in the medium revealed to be reproducible which indicates a non-interfering behaviour of the hydrogel under the conditions tested here.

\section{Discussion}

The system presented in this study is suitable to be used for in vitro release studies. It demonstrates the possibility to test the release of dexamethasone without the DDS being completely surrounded by medium. The chosen time points and the measured concentrations further indicate that an influence of the hydrogel on the release is rather negligible. Similar results were found by Seidlitz et al. who tested the distribution of sodium fluorescein and triamterene through such a hydrogel. [3] Therein, the concentration of the substances in the release medium, the stent coating and the hydrogel did not change after $24 \mathrm{~h} \mathrm{[3].} \mathrm{Therefore,} \mathrm{if} \mathrm{alginate}$ possesses binding sites for dexamethasone, these are probably saturated at early time points, leading to no measurable interferences during the study. However, dexamethasone bound to the hydrogel was not quantified and remains to be investigated, also in dependence to the spatial dimensions of the hydrogel. To assess the clinical relevance of the concentrations measured with the reported test system, several additional parameters need to be taken into account, such as perilymph volume, diffusion characteristics of dexamethasone through the round window membrane, and the effective concentration of dexamethasone in the cochlear perilymph. Drug concentrations observed in this study revealed that no sink conditions were ensured. This was expected, because the drug loading of the samples was chosen to be high in order to overcome possible adverse effects, such as hydrogel binding of the drug.

The system introduced here could be used to repeatedly aspirate and refill the medium. Anyway the production of the system had to be repeated every once in a while due to the hitherto relatively low mechanical stability of the hydrogel and mechanical stress applied by the repeated insertion of the cannula for the medium change. Except for mechanical instability, no other stability problems were observed.

\section{Conclusion}

An alginate based quasi-stationary test system for in vitro drug release studies was successfully designed and manufactured. The system is easy to build and therefore suitable to monitor the drug release of dexamethasone from PEGDA $_{700}$ derived polymer samples over a certain period of time. Further challenges that remain to be considered are the low mechanical stability of the hydrogel, which results in the need for frequent manufacturing during the handling of the system. Therefore, the use of other hydrogel forming 
substances as well as testing the drug release from samples with lower drug incorporation, patient relevant geometries under sink conditions will be subject to future studies.

Acknowledgment: The authors would like to thank Natalie Kira Kietz, Caroline Dudda and Andrea Rohde for the excellent technical assistance.

\section{Author Statement}

Research funding: Financial support by the Federal Ministry of Education and Research (BMBF) within RESPONSE "Partnership for Innovation in Implant Technology" is gratefully acknowledged. Conflict of interest: Authors state no conflict of interest. Informed consent: Informed consent is not applicable. Ethical approval: The conducted research is not related to either human or animal use.

\section{References}

[1] Krenzlin S, Vincent C, Munzke L, Gnansia D, Siepmann F. Predictability of drug release from cochlear implants. Journal of Controlled Release. 2012;159:60-68.

[2] Bohl A, Rohm HW, Ceschi P, Paasche G, Hahn A, Barcikowski S, Lenarz T, Stöver T, Pau HW, Schmitz KP Sternberg K. Development of a specially tailored local drug delivery system for the prevention of fibrosis after insertion of cochlear implants into the inner ear J Mater Sci: Mater Med 2012;23:2151-2162

[3] Seidlitz A, Nagel S, Semmling B, Grabow N, Martin H, Senz V, Harder C, Sternberg K, Schmitz KB, Kroemer H,

Weitschies W. Examination of drug release and distribution from drug-eluting stents with a vessel-simulating flow-through cell. European Journal of Pharmaceutics and Biopharmaceutics 2011;78:36-48 